\title{
Cardiomiopatia de Takotsubo como diagnóstico diferencial de síndrome coronariana aguda: diferenças entre os aspectos clínicos
}

Takotsubo cardiomyopathy as a differential diagnosis of acute coronary syndrome: differences between clinical features

Miocardiopatía de Takotsubo como diagnóstico diferencial del síndrome coronario agudo: diferencias entre características clínicas

Isabella Pires Gomes Mendes ORCID: https://orcid.org/0000-0003-3486-7908 Universidade Federal do Delta do Parnaíba, Brasil

E-mail: isabellapiresmendes@gmail.com

Olívio Joaquim Fonseca Neto ORCID: https://orcid.org/0000-0002-2695-0077 Universidade Federal do Delta do Parnaíba, Brasil

E-mail: oliviofonsecaneto@ hotmail.com

Breno Vítor Rodrigues Coqueiro Santana

ORCID: https://orcid.org/0000-0001-6286-9607

Universidade Federal do Delta do Parnaíba, Brasil E-mail: brenosantana@ufpi.edu.br

Juliano Luiz de Souza

ORCID: https://orcid.org/0000-0003-0567-2167

Universidade Federal do Delta do Parnaíba, Brasil

E-mail: julianoluizdesouza@ufpi.edu.br

Brenda Larissa Andrade Viana

ORCID: https://orcid.org/0000-0003-2285-4309

Universidade Federal do Delta do Parnaíba, Brasil

E-mail: brendalarissa@ufpi.edu.br

Rafael Santos Correia

ORCID: https://orcid.org/0000-0002-5711-7838

Universidade Federal do Delta do Parnaíba, Brasil

E-mail: rafael0094@ufpi.edu.br

Heliton José Baquil Araujo

ORCID: https://orcid.org/0000-0002-9257-5885 Instituto de Educação Superior do Vale do Parnaíba, Brasil

E-mail: heliton_jose@hotmail.com

Yasser da Silveira Krüger

ORCID: https://orcid.org/0000-0002-6163-7553

Universidade Federal do Delta do Parnaíba, Brasil

E-mail: yasser.kruger@gmail.com

Érica de Araújo Silva Mendes

ORCID: https://orcid.org/0000-0001-5003-037X

Universidade Federal do Delta do Parnaíba, Brasil

E-mail: ericasilva.ma@gmail.com

Antonino Neto Coelho Moita

ORCID: https://orcid.org/0000-0002-4993-2568

Universidade Federal do Delta do Parnaíba, Brasil

E-mail: antoninomoita@ufpi.edu.br

\begin{abstract}
Resumo
Objetivo: Demonstrar a importância de ter a Cardiomiopatia de Takotsubo (CT) como diagnóstico diferencial para síndrome coronariana aguda (SCA), através da análise dos aspectos clínicos dessa condição. Metodologia: Revisão integrativa de literatura que buscou responder a pergunta "Como é feito o diagnóstico dos pacientes com cardiomiopatia de Takotsubo, diferenciando da Síndrome Coronariana Aguda, a partir da apresentação clínica?”, desenvolvida através da estratégia PICo e cujos artigos foram pesquisados nas bases de dados: Medical Literature and Retrieval System onLine (MEDLINE/PubMed®) via National Library of Medicine; Scientific Electronic Library Online (SciELO) e Literatura Latino-Americana e do Caribe em Ciências da Saúde (LILACS). Resultados: Os pacientes com CT eram predominantemente mulheres, com idade média entre 60 a 70 anos em mais de $90 \%$ dos casos
\end{abstract}


e apresentavam semelhanças à SCA, o que se apresenta como um desafio na diferenciação entre as duas. Os quadros clínicos se assemelham pela presença de dor torácica e dispneia, incluindo alterações no ECG e nos biomarcadores cardíacos, além de poderem apresentar dados de mortalidade e complicações intra-hospitalares semelhantes. As alterações eletrocardiográficas, embora presente em ambas as condições, apresentam padrões distintos. Conclusão: As duas afecções, a cardiomiopatia de Takotsubo e a Síndrome Coronariana Aguda, possuem apresentações clínicas bastante semelhantes, devendo a primeira ser investigada como diagnóstico diferencial da segunda. Para a diferenciação das duas e confirmação diagnóstica da cardiomiopatia de Takotsubo, os critérios de Mayo devem ser contemplados, através do auxílio dos exames complementares eletrocardiograma e ecocardiografia.

Palavras-chave: Cardiomiopatia de Takotsubo; Síndrome coronariana aguda; Diagnóstico diferencial; Manifestações clínicas.

\begin{abstract}
Objective: To demonstrate the importance of having Takotsubo Cardiomyopathy (TC) as a differential diagnosis for acute coronary syndrome (ACS), through the analysis of the clinical aspects of this condition. Methods: Integrative literature review that sought to answer the question "How is the diagnosis of patients with Takotsubo cardiomyopathy, differentiating from Acute Coronary Syndrome, based on the clinical presentation?", developed through the PICo strategy and whose articles were searched in the databases sources: Medical Literature and Retrieval System onLine (MEDLINE/PubMed®) via National Library of Medicine; Scientific Electronic Library Online (SciELO) and Literatura Latino-Americana e do Caribe em Ciências da Saúde (LILACS). Results: Patients with TC were predominantly women, with a mean age between 60 and 70 years in more than $90 \%$ of the cases and they presented similarities to ACS, which presents a challenge in differentiating the two. The clinical pictures are similar due to the presence of chest pain and dyspnea, including changes in the ECG and cardiac biomarkers, in addition to presenting similar data on mortality and in-hospital complications. Electrocardiographic alterations, although present in both conditions, present different patterns. Conclusion: The two conditions, TC and ACS, have very similar clinical presentations, and the first should be investigated as a differential diagnosis of the second. For the differentiation of the two and diagnostic confirmation of Takotsubo cardiomyopathy, the Mayo criteria must be considered, through the help of complementary exams, such as electrocardiogram and echocardiography.
\end{abstract}

Keywords: Takotsubo cardiomyopathy; Acute coronary syndrome; Differential diagnosis; Clinical manifestations.

\title{
Resumen
}

Objetivo: Demostrar la importancia de la Miocardiopatía de Takotsubo (TC) como diagnóstico diferencial del síndrome coronario agudo (SCA), a través del análisis de los aspectos clínicos de esta patología. Metodología: Revisión de la literatura integradora que buscó dar respuesta a la pregunta "¿Cómo se realiza el diagnóstico de pacientes con miocardiopatía de Takotsubo, diferenciando el Síndrome Coronario Agudo, de la presentación clínica?", Desarrollado a través de la estrategia PICo y cuyos artículos se buscaron en las fuentes de las bases de datos. : Sistema de Recuperación y Literatura Médica en Línea (MEDLINE / PubMed®) a través de la Biblioteca Nacional de Medicina; Biblioteca Electrónica Científica en Línea (SciELO) y Literatura de Ciencias de la Salud de América Latina y el Caribe (LILACS). Resultados: Los pacientes con CT eran predominantemente mujeres, con una edad media entre 60 y 70 años en más del 90\% de los casos y tenían similitudes con el SCA, representando un desafío para diferenciar los dos. Los cuadros clínicos son similares por la presencia de dolor torácico y disnea, incluyendo cambios en el ECG y biomarcadores cardíacos, además de presentar datos similares sobre mortalidad y complicaciones intrahospitalarias. Las alteraciones electrocardiográficas, aunque presentes en ambas condiciones, presentan patrones diferentes. Conclusión: Las dos condiciones, miocardiopatía de Takotsubo y síndrome coronario agudo, tienen presentaciones clínicas muy similares, y la primera debe ser investigada como diagnóstico diferencial de la segunda. Para la diferenciación de los dos y la confirmación diagnóstica de la miocardiopatía de Takotsubo, se deben considerar los criterios de Mayo, mediante la ayuda de los exámenes complementarios electrocardiograma y ecocardiografía.

Palabras clave: Miocardiopatía de Takotsubo; Síndrome coronario agudo; Diagnóstico diferencial; Manifestaciones clínicas.

\section{Introdução}

A cardiomiopatia induzida por estresse ou síndrome do coração partido é uma disfunção aguda e reversível do ventrículo esquerdo descrita pela primeira vez em 1990, no Japão, sendo denominada, a partir de então, de Cardiomiopatia de Takotsubo (CT), passando a ter, assim, uma maior relevância na comunidade científica e posteriormente sendo descrita na Europa, América do Norte e Austrália (Lee et al., 2017).

Essa patologia é esperada entre $1 \%$ a $2 \%$ dentre os casos suspeitos de Infarto Agudo do Miocárdio (IAM), possuindo um acometimento clínico similar à síndrome coronariana aguda (SCA) (Kim et al., 2018; Samardhie et al., 2011). Apesar das 
similaridades, entretanto, há diferenças na apresentação clínica e nos achados de exames complementares entre as doenças (Samardhie et al., 2011).

Há indícios de que a CT pode ser predisposta por estressores físicos extremos emocionais e não neurológicos em mulheres na pós-menopausa, o que sugere atuação do sistema nervoso simpático, sendo um fator valioso na diferenciação entre as duas síndromes. Entretanto, pode ocorrer na ausência de um estressor em até um terço dos casos (Kim et al., 2018). Nesse contexto, os achados clínicos de pacientes com CT podem ser a chave para o diagnóstico correto da síndrome, incluindo ausência de acometimento das coronárias e presença de acinesia apical, assim como hipercinesia basal do ventrículo esquerdo (Reis \& Rosas, 2010).

Por se tratar de uma doença não convencional, transitória, reversível e de difícil diagnóstico, necessita-se de uma maior visibilidade e produções acadêmicas sobre o tema, facilitando diagnósticos e condutas de avaliação (Fröhlich et al., 2011; Lee et al., 2017). Diante disso, temos como objetivo demonstrar a importância de ter a CT como diagnóstico diferencial para SCA, através da análise dos aspectos clínicos dessa condição.

\section{Metodologia}

O presente estudo é uma revisão integrativa de literatura, uma das metodologias de revisão mais abrangente, pois permite ao pesquisador definir conceitos, revisar teorias e evidências e analisar problemas metodológicos referentes a um determinado tema (Souza et al., 2010). Essa abordagem é considerada como um dos primeiros passos para a iniciação científica, já que permite organização e discussão de um determinado assunto, possibilitando o surgimento de novas teorias e o reconhecimento de lacunas, sendo, assim, importante para a construção do conhecimento científico (Botelho et al.,2011).

$\mathrm{Na}$ área da saúde, a revisão integrativa de literatura é um recurso valioso à medida que permite aos profissionais um melhor aproveitamento de evidências de vários estudos (Souza et al., 2010). Segundo Souza et al. (2010), isso é possível pelo fato dessa abordagem apresentar etapas metodológicas sucintas ao abordar uma alta quantidade e complexidade de informações presentes na área da saúde, incluindo diversos tipos de estudos, experimentais e não experimentais, além de combinar dados da literatura teórica e empírica. Assim, a revisão integrativa tem o poder de fornecer síntese de conhecimento e aplicabilidade de resultados na prática (Souza et al., 2010).

Segundo Botelho et al. (2011), a construção de uma revisão integrativa deve ser feita a partir de uma sucessão de etapas bem definidas, que foram seguidas para a realização do presente estudo: (1) identificação do tema e seleção da questão de pesquisa, que conta com a definição de um problema e a formulação de uma pergunta de pesquisa; (2) estabelecimento de critérios de inclusão e exclusão, para nortear a busca nas bases de dados; (3) identificação dos estudos pré-selecionados e selecionados, através da leitura dos títulos, resumos e palavras-chave de todas as publicações encontradas pela estratégia de busca, além da verificação dos critérios de inclusão; (4) categorização dos estudos selecionados, com a finalidade de sumarizar as informações relevantes dos artigos encontrados; (5) análise e interpretação dos resultados, a partir da discussão dos mesmos; e (6) apresentação da revisão/síntese do conhecimento.

Quanto à questão de pesquisa, o presente estudo buscou responder a pergunta "Como é feito o diagnóstico dos pacientes com cardiomiopatia de Takotsubo, diferenciando da Síndrome Coronariana Aguda, a partir da apresentação clínica?", desenvolvida através da estratégia PICo (Quadro 1), que corresponde a um acrônimo para Paciente/População/Problema, Interesse e Contexto. Esses elementos são essenciais para a questão de pesquisa em estudos não-clínicos e são bastante utilizados para tratar de problemas que emergem da prática assistencial, ensino ou pesquisa (Santos et al., 2007; Takahashi et al., 2014). 
Quadro 1 - Pergunta de pesquisa segundo a estratégia PICo. Parnaíba, PI, Brasil 2021.

\begin{tabular}{|c|c|c|c|c|}
\hline DESCRIÇÃO & PICo & COMPONENTES & DESCRITORES & TIPO \\
\hline $\begin{array}{c}\text { População/ } \\
\text { Problema }\end{array}$ & $\mathrm{P}$ & Cardiomiopatia de Takotsubo & $\begin{array}{c}\text { "takotsubo cardiomyopathy" } \\
\text { "broken heart syndrome" } \\
\text { "takotsubo syndrome" }\end{array}$ & DeCS/ MeSH \\
\hline Interesse & $\mathrm{I}$ & Apresentação clínica & "ventricular dysfunction" "cardiac \\
stress" & $\begin{array}{c}\text { DeCS/ } \\
M e S H\end{array}$ \\
\hline Contexto & Co & Diagnóstico & "diagnosis" & DeCS/ \\
\hline
\end{tabular}

Fonte: Autores.

Para a seleção dos artigos foram utilizadas as bases de dados: Medical Literature and Retrieval System onLine (MEDLINE/PubMed®) via National Library of Medicine; Scientific Electronic Library Online (SciELO) e Literatura LatinoAmericana e do Caribe em Ciências da Saúde (LILACS). Os descritores controlados, utilizados na estratégia de busca, foram selecionados no MESH (Medical Subject Headings) e DeCs (Descritores em Ciências da Saúde). Em todas as bases de dados foi utilizada a mesma estratégia de busca (Quadro 2).

Quadro 2 - Estratégia de busca utilizada nas bases de dados PubMed, SciELO e LILACS. Parnaíba, PI, Brasil, 2021.

\begin{tabular}{|c|l|c|c|}
\hline $\begin{array}{c}\text { BASES DE } \\
\text { DADOS }\end{array}$ & \multicolumn{1}{|c|}{ ESTRATÉGIA DE BUSCA } & RESULTADOS & SELECIONADOS \\
\hline PubMed & $\begin{array}{l}\text { (takotsubo cardiomyopathy OR broken heart syndrome OR takotsubo } \\
\text { syndrome) AND diagnosis AND ventricular dysfunction AND cardiac } \\
\text { stress }\end{array}$ & 626 & 29 \\
\hline SciELO & $\begin{array}{l}\text { (takotsubo cardiomyopathy OR broken heart syndrome OR takotsubo } \\
\text { syndrome) AND diagnosis AND ventricular dysfunction AND cardiac } \\
\text { stress }\end{array}$ & 1 & 0 \\
\hline LILACS & $\begin{array}{l}\text { (takotsubo cardiomyopathy OR broken heart syndrome OR takotsubo } \\
\text { syndrome) AND diagnosis AND ventricular dysfunction AND cardiac } \\
\text { stress }\end{array}$ & 13 & 0 \\
\hline
\end{tabular}

Fonte: Autores.

Foram incluídos no estudo apenas artigos originais, disponíveis online na íntegra nas bases de dados selecionadas e que foram publicados nos últimos 10 anos, com recorte temporal dos anos de 2011 a 2021. Foram excluídos os estudos não observacionais: artigos de revisão, teses, dissertações e materiais não científicos; os estudos observacionais com testes em não humanos; artigos em que não foi possível identificar relação com a temática por meio da leitura de título e resumo e aqueles que estiverem duplicados nas bases de dados, além dos estudos publicados em outros idiomas que não português, inglês e espanhol.

Essa busca e seleção dos artigos foi realizada por um revisor. A priori, a seleção dos estudos se deu pela leitura dos títulos e resumos, com base nos critérios de inclusão. Posteriormente, os artigos restantes foram lidos na íntegra, a fim de incluir apenas as publicações relevantes e coerentes com o problema deste estudo. Por fim, para a apresentação da revisão, foi feita análise e discussão para interpretação dos resultados dos estudos selecionados, por todos os pesquisadores. 


\section{Resultados}

De 640 artigos identificados nas bases de dados, 106 estudos foram selecionados para análise, após eliminação de artigos duplicados nas bases ou que se enquadraram nos critérios de exclusão, conforme fluxograma (Figura 1).

Figura 1 - Fluxograma representativo do processo de seleção dos artigos na revisão integrativa, Brasil, 2021.

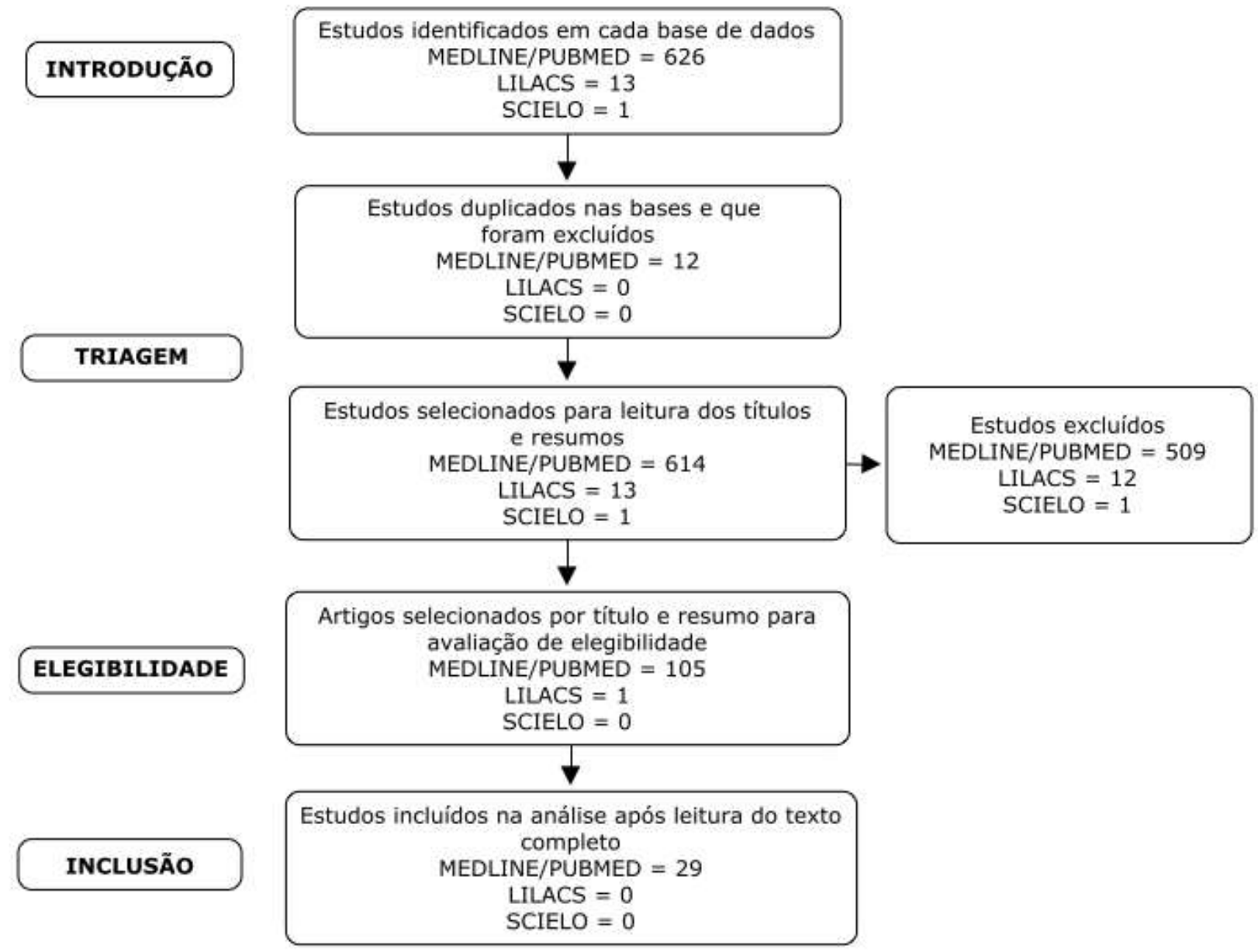

Fonte: Autores.

Para compor a revisão integrativa, foram incluídos 29 artigos. Dentre os artigos selecionados, a maioria foi publicada no ano de $2016(n=5 ; 17,2 \%)$, seguido de 2011, 2014 e $2015(n=4 ; 13,8 \%$, em cada ano), de 2019 ( $n=3 ; 10,3 \%), 2013,2017,2018$ e $2020(n=2 ; 6,9 \%$, em cada ano) e $2012(n=1 ; 3,5 \%)$. Todos os estudos foram publicados em periódicos internacionais e os países com maior número de publicações foram Estados Unidos, Suíça e Alemanha com 10 (35,5\%), 7 (24,1\%) e 3 (10,3\%) publicações cada, respectivamente. A tabela 01 detalha os artigos que compuseram este estudo. 
Tabela 1 - Caracterização dos artigos quanto ao título, autor/ano, objetivo, local de realização e local de publicação. Brasil, 2021.

\begin{tabular}{|c|c|c|c|c|}
\hline TÍTULO & AUTORES/ANO & OBJETIVO & $\begin{array}{l}\text { LOCAL DE } \\
\text { REALIZAÇ } \\
\quad \tilde{A} O\end{array}$ & $\begin{array}{l}\text { LOCAL DE } \\
\text { PUBLICAÇÃO }\end{array}$ \\
\hline $\begin{array}{l}\text { Characterization of Tako-tsubo } \\
\text { Cardiomyopathy in Spain: Results } \\
\text { from the RETAKO National } \\
\text { Registry }\end{array}$ & $\begin{array}{l}\text { Núñez Gil et al. } \\
\qquad(2015)\end{array}$ & $\begin{array}{c}\text { Criar um registro nacional multicêntrico colaborativo } \\
\text { de casos de cardiomiopatia de Takotsubo, a fim de } \\
\text { descrever esta } \\
\text { doença com mais detalhes em nosso meio }\end{array}$ & Espanha & $\begin{array}{l}\text { Revista Española de } \\
\text { Cardiología }\end{array}$ \\
\hline $\begin{array}{l}\text { Brain natriuretic peptide in apical } \\
\text { ballooning syndrome } \\
\text { (Takotsubo/stress cardiomyopathy): } \\
\text { comparison with acute } \\
\text { myocardial infarction }\end{array}$ & $\begin{array}{l}\text { Ahmeda, } \\
\text { Madhavanb \& } \\
\text { Prasadb, (2012) }\end{array}$ & $\begin{array}{l}\text { Determinar as correlações clínicas e hemodinâmicas } \\
\text { dos níveis de peptídeo natriurético cerebral na } \\
\text { Síndrome do Balonismo Apical e comparar os perfis } \\
\text { de biomarcadores na Síndrome do Balonismo Apical } \\
\text { com grupo controle de Infarto Agudo do Miocárdio }\end{array}$ & $\begin{array}{l}\text { Estados } \\
\text { Unidos }\end{array}$ & $\begin{array}{l}\text { Coronary Artery } \\
\text { Disease }\end{array}$ \\
\hline $\begin{array}{l}\text { Myocardial strain may be useful in } \\
\text { differentiating Takotsubo } \\
\text { cardiomyopathy from left anterior } \\
\text { descending coronary artery } \\
\text { ischemia. }\end{array}$ & $\begin{array}{l}\text { Cai, Addetia, } \\
\text { Medvedofsky \& } \\
\text { Spencer (2017) }\end{array}$ & $\begin{array}{c}\text { Avaliar sistematicamente a função regional dos } \\
\text { ventrículos esquerdo e direito na síndrome de } \\
\text { Takotsubo com imagem de deformação do miocárdio } \\
\text { para avaliar } \\
\text { se o envolvimento ventricular pode diferir entre } \\
\text { síndrome de Takotsubo e isquemia da coronária } \\
\text { descendente anterior. }\end{array}$ & $\begin{array}{l}\text { Estados } \\
\text { Unidos }\end{array}$ & $\begin{array}{l}\text { International Journal of } \\
\text { Cardiology }\end{array}$ \\
\hline $\begin{array}{c}\text { Clinical Features of Takotsubo } \\
\text { Cardiomyopathy - A Single-Center } \\
\text { Experience }\end{array}$ & $\begin{array}{c}\text { Dias, Franco, } \\
\text { Mercedes, Hebert, } \\
\text { Messina, \& Quevedo } \\
\text { (2013) }\end{array}$ & $\begin{array}{c}\text { Avaliar as características clínicas e prognósticos de } 78 \\
\text { pacientes que tiveram síndrome de Takotsubo }\end{array}$ & $\begin{array}{l}\text { Estados } \\
\text { Unidos }\end{array}$ & Cardiology \\
\hline $\begin{array}{l}\text { Clinical Characteristics and } \\
\text { Cardiovascular Magnetic Resonance } \\
\text { Findings in Stress (Takotsubo) } \\
\text { Cardiomyopathy }\end{array}$ & Eitel et al. (2011) & $\begin{array}{l}\text { Definir de forma abrangente o espectro clínico e a } \\
\text { evolução da cardiomiopatia de estresse em uma } \\
\text { grande população, incluindo dados de caracterização } \\
\text { de tecido a partir de imagem de ressonância } \\
\text { magnética; e estabelecer um conjunto de critérios } \\
\text { adequados para tomada de decisão diagnóstica em } \\
\text { pacientes que se apresentam agudamente com } \\
\text { suspeita de cardiomiopatia de estresse }\end{array}$ & Alemanha & $\begin{array}{l}\text { Journal of the } \\
\text { American Medical } \\
\text { Association (JAMA) }\end{array}$ \\
\hline $\begin{array}{c}\text { ECG Criteria to Differentiate } \\
\text { Between Takotsubo (Stress) } \\
\text { Cardiomyopathy and Myocardial } \\
\text { Infarction }\end{array}$ & $\begin{array}{l}\text { Frangieh et al. } \\
\quad(2016)\end{array}$ & $\begin{array}{l}\text { Comparar o Eletrocardiograma (ECG) de admissão } \\
\text { entre síndrome de Takotsubo e Infarto Agudo do } \\
\text { Miocárdio (IAM), incluindo IAM com elevação de } \\
\text { segmento ST e IAM sem supradesnivelamento do } \\
\text { segmento ST, a fim de definir alterações específicas } \\
\text { de ECG para diferenciar as duas entidades }\end{array}$ & Suíça & $\begin{array}{c}\text { Journal of the } \\
\text { American Heart } \\
\text { Association (JAHA) }\end{array}$ \\
\hline $\begin{array}{l}\text { Takotsubo cardiomyopathy has a } \\
\text { unique cardiac biomarker profile: } \\
\text { NT-proBNP/myoglobin and NT- } \\
\text { proBNP/troponin T ratios for the } \\
\text { differential diagnosis of acute } \\
\text { coronary syndromes and stress } \\
\text { induced cardiomyopathy }\end{array}$ & Fröhlich et al. (2011) & $\begin{array}{l}\text { Identificar síndrome de Takotsubo por seu } \\
\text { perfil único de biomarcador cardíaco em um estágio } \\
\text { inicial e, de preferência, apenas com procedimentos } \\
\text { não invasivos }\end{array}$ & Suíça & $\begin{array}{l}\text { International Journal of } \\
\text { Cardiology }\end{array}$ \\
\hline $\begin{array}{l}\text { A novel clinical score (InterTAK } \\
\text { Diagnostic } \\
\text { Score) to differentiate takotsubo } \\
\text { syndrome from acute coronary } \\
\text { syndrome: results from the } \\
\text { InternationalTakotsubo Registry }\end{array}$ & Ghadri et al. (2016) & $\begin{array}{c}\text { Desenvolver uma pontuação clínica para estimar a } \\
\text { probabilidade de síndrome de Takotsubo e distingui- } \\
\text { la de síndrome coronariana na fase aguda }\end{array}$ & Suíça & $\begin{array}{l}\text { European Journal of } \\
\text { Heart Failure }\end{array}$ \\
\hline $\begin{array}{l}\text { Long-Term Prognosis of Patients } \\
\text { With Takotsubo Syndrome }\end{array}$ & Ghadri et al. (2018) & $\begin{array}{l}\text { Comparar o prognóstico entre pacientes com } \\
\text { síndrome coronariana aguda e a síndrome de } \\
\text { Takotsubo e investigarprognóstico de curto e longo } \\
\text { prazo na síndrome de Takotsubo com base em } \\
\text { diferentes gatilhos }\end{array}$ & Suíça & $\begin{array}{l}\text { Journal of the } \\
\text { American College of } \\
\text { Cardiology }\end{array}$ \\
\hline $\begin{array}{l}\text { A signature of circulating } \\
\text { microRNAs differentiates takotsubo } \\
\text { cardiomyopathy from acute } \\
\text { myocardial infarction }\end{array}$ & $\begin{array}{l}\text { Jaguszewski et al. } \\
\qquad(2014)\end{array}$ & $\begin{array}{c}\text { Identificar microRNAs circulantes adequados para o } \\
\text { diagnóstico de cardiomiopatia de Takotsubo aguda e } \\
\text { para distingui-la de Infarto Agudo do Miocárdio }\end{array}$ & Suíça & $\begin{array}{l}\text { European Heart } \\
\text { Journal }\end{array}$ \\
\hline
\end{tabular}




\begin{tabular}{|c|c|c|c|c|}
\hline $\begin{array}{l}\text { Natural history and predictors of } \\
\text { mortality of patients with Takotsubo } \\
\text { syndrome }\end{array}$ & Kim et al. (2018) & $\begin{array}{c}\text { Avaliar a história natural e os determinantes dos } \\
\text { prognósticos em pacientes com síndrome de } \\
\text { Takotsubo }\end{array}$ & $\begin{array}{l}\text { Estados } \\
\text { Unidos }\end{array}$ & $\begin{array}{l}\text { International Journal o } \\
\text { Cardiology }\end{array}$ \\
\hline $\begin{array}{l}\text { Takotsubo cardiomyopathy: ten year } \\
\text { experience at a tertiary care hospital } \\
\text { in Pakistan }\end{array}$ & $\begin{array}{l}\text { Laghari, Khan \& } \\
\text { Kazmi (2014) }\end{array}$ & $\begin{array}{c}\text { Determinar os dados demográficos, apresentações } \\
\text { clínicas, complicações } \\
\text { e recuperação da função sistólica do ventrículo } \\
\text { esquerdo em pacientes com síndrome de Takotsubo } \\
\text { de origem paquistanesa }\end{array}$ & Paquistão & BMC Research Notes \\
\hline $\begin{array}{l}\text { Discrimination of stress (Takotsubo) } \\
\text { cardiomyopathy from acute } \\
\text { coronary syndrome with clinical risk } \\
\text { factors and coronary evaluation in } \\
\text { real-world clinical practice }\end{array}$ & Lee et al. (2017) & $\begin{array}{c}\text { Investigar a frequência de avaliação coronariana que } \\
\text { tem sido feita em pacientes com suspeita de síndrome } \\
\text { de Takotsubo e destacar os fatores associados à } \\
\text { tomada de decisão para realizar ou não uma avaliação } \\
\text { coronariana }\end{array}$ & Coreia do Sul & $\begin{array}{c}\text { International Journal o } \\
\text { Cardiology }\end{array}$ \\
\hline $\begin{array}{l}\text { A Score to differentiate Takotsubo } \\
\text { syndrome from non-ST-elevation } \\
\text { myocardial infarction in women at } \\
\text { the bedside }\end{array}$ & Looi et al. (2020) & $\begin{array}{l}\text { Validar uma pontuação simples usando } \\
\text { dados demográficos, clínicos e de ECG para } \\
\text { distinguir mulheres com infarto do miocárdio sem } \\
\text { supradesnivelamento do segmento ST de síndrome de } \\
\text { Takotsubo sem supradesnivelamento do segmento ST }\end{array}$ & $\begin{array}{c}\text { Nova } \\
\text { Zelândia }\end{array}$ & Open Heart \\
\hline $\begin{array}{l}\text { Systolic and Diastolic Mechanics in } \\
\text { Stress Cardiomyopathy }\end{array}$ & $\begin{array}{l}\text { Medeiros et al. } \\
\quad(2014)\end{array}$ & $\begin{array}{l}\text { Analisar se as propriedades sistólicas e diastólicas de } \\
\text { pacientes com cardiomiopatia de estresse seriam } \\
\text { fáceis de diferenciar daquelas dos pacientes com } \\
\text { Infarto Agudo do Miocárdio da coronária descendente } \\
\text { anterior }\end{array}$ & $\begin{array}{l}\text { Estados } \\
\text { Unidos }\end{array}$ & Circulation \\
\hline $\begin{array}{l}\text { Endogenous stress response in } \\
\text { Takotsubo } \\
\text { cardiomyopathy and acute } \\
\text { myocardial infarction }\end{array}$ & $\begin{array}{l}\text { Meissner et al. } \\
\quad \text { (2011) }\end{array}$ & $\begin{array}{l}\text { Examinar a resposta ao estresse endógeno na } \\
\text { apresentação, quantificada pelo nível de copeptina, de } \\
\text { pacientes com cardiomiopatia de Takotsubo e } \\
\text { pacientes com Infarto Agudo do Miocárdio }\end{array}$ & $\begin{array}{l}\text { Alemanha e } \\
\text { Suíça }\end{array}$ & $\begin{array}{l}\text { European Journal of } \\
\text { Clinical Investigation }\end{array}$ \\
\hline $\begin{array}{l}\text { Clinical Outcome of Takotsubo } \\
\text { Cardiomyopathy Diagnosed With or } \\
\text { Without Coronary Angiography }\end{array}$ & $\begin{array}{l}\text { Misumida et al. } \\
\text { (2019) }\end{array}$ & $\begin{array}{c}\text { Esclarecer a taxa de angiografia coronária em } \\
\text { pacientes com diagnóstico de Síndrome de Takotsubo } \\
\text { (ST) e também examinar o prognóstico de pacientes } \\
\text { diagnosticados com ST com e sem } \\
\text { angiografia coronária }\end{array}$ & $\begin{array}{l}\text { Estados } \\
\text { Unidos }\end{array}$ & Angiology \\
\hline $\begin{array}{l}\text { Usefulness of the Troponin-Ejection } \\
\text { Fraction Product to Differentiate } \\
\text { Stress Cardiomyopathy from ST- } \\
\text { Segment Elevation Myocardial } \\
\text { Infarction }\end{array}$ & $\begin{array}{l}\text { Nascimento et al. } \\
\text { (2014) }\end{array}$ & $\begin{array}{c}\text { Desenvolver uma ferramenta clínica não invasiva para } \\
\text { discriminar entre Infarto do Miocárdio com } \\
\text { supradesnivelamento do segmento ST e a } \\
\text { cardiomiopatia de estresse }\end{array}$ & $\begin{array}{r}\text { Estados } \\
\text { Unidos }\end{array}$ & $\begin{array}{l}\text { The American Journal } \\
\text { of Cardiology }\end{array}$ \\
\hline $\begin{array}{l}\text { Takotsubo cardiomyopathy: an } \\
\text { Australian single centre } \\
\text { experience with medium term } \\
\text { follow up }\end{array}$ & $\begin{array}{l}\text { Samardhieet al. } \\
\text { (2011) }\end{array}$ & $\begin{array}{c}\text { Apresentar os dados demográficos, características } \\
\text { clínicas e dados de acompanhamento de médio prazo } \\
\text { de um } \\
\text { grande experiência em um único centro na Austrália }\end{array}$ & Austrália & $\begin{array}{l}\text { Internal Medicine } \\
\text { Journal }\end{array}$ \\
\hline $\begin{array}{l}\text { Assessment of the German and } \\
\text { Italian Stress Cardiomyopathy } \\
\text { Score for Risk Stratification for In- } \\
\text { hospital Complications } \\
\text { in Patients With Takotsubo } \\
\text { Syndrome }\end{array}$ & Santoro et al. (2019) & $\begin{array}{c}\text { Derivar uma pontuação clínica simples para previsão } \\
\text { de risco de complicações hospitalares entre pacientes } \\
\text { com síndrome de Takotsubo. }\end{array}$ & Itália & JAMA Cardiology \\
\hline $\begin{array}{l}\text { Alterations in Cardiac Deformation, } \\
\text { Timing of Contraction and } \\
\text { Relaxation, and Early Myocardial } \\
\text { Fibrosis Accompany the Apparent } \\
\text { Recovery of Acute Stress-Induced } \\
\text { (Takotsubo) Cardiomyopathy: An } \\
\text { End to the Concept of Transience }\end{array}$ & Schwars et al. (2017) & $\begin{array}{l}\text { Testar a hipótese de que a restauração da fração de } \\
\text { ejeção normal após a síndrome de Takotsubo aguda } \\
\text { não é equivalente à recuperação funcional completa }\end{array}$ & Reino Unido & $\begin{array}{l}\text { Journal of the } \\
\text { American Society of } \\
\text { Echocardiography }\end{array}$ \\
\hline $\begin{array}{l}\text { Long-term excess mortality in } \\
\text { takotsubo cardiomyopathy: } \\
\text { predictors, causes and clinical } \\
\text { consequences }\end{array}$ & $\begin{array}{l}\text { Stiermaieret al. } \\
\quad(2016)\end{array}$ & $\begin{array}{l}\text { Comparar a taxa de mortalidade a longo prazo de } \\
\text { pacientes com síndrome de Takotsubo com pacientes } \\
\text { de alto risco apresentando infarto do miocárdio com } \\
\text { elevação do segmento ST }\end{array}$ & Alemanha & $\begin{array}{l}\text { European Journal of } \\
\text { Heart Failure }\end{array}$ \\
\hline $\begin{array}{l}\text { Clinical Features and Outcomes of } \\
\text { Takotsubo (Stress) Cardiomyopathy }\end{array}$ & Templin et al. (2015) & $\begin{array}{c}\text { Investigar as características clínicas, definir preditores } \\
\text { de prognóstico e avaliar o curso clínico e o resultado } \\
\text { de cardiomiopatia de Takotsubo }\end{array}$ & Suíça & $\begin{array}{l}\text { The New England } \\
\text { Journal of Medicine }\end{array}$ \\
\hline
\end{tabular}




\begin{tabular}{|c|c|c|c|c|}
\hline $\begin{array}{l}\text { Cardiogenic Shock in Takotsubo } \\
\text { Cardiomyopathy Versus Acute } \\
\text { Myocardial Infarction: An 8-Year } \\
\text { National Perspective on Clinical } \\
\text { Characteristics, Management, and } \\
\text { Outcomes }\end{array}$ & $\begin{array}{l}\text { Vallabhajosyula et } \\
\text { al. (2019) }\end{array}$ & $\begin{array}{l}\text { Comparar as características clínicas e } \\
\text { resultados de choque cardiogênico na cardiomiopatia } \\
\text { de Takotsuba para aqueles de choque cardiogênico no } \\
\text { infarto miocárdico entre pacientes hospitalizados nos } \\
\text { Estados Unidos }\end{array}$ & $\begin{array}{l}\text { Estados } \\
\text { Unidos }\end{array}$ & Heart Failure \\
\hline $\begin{array}{l}\text { Comparison of Complications and } \\
\text { In-Hospital Mortality in Takotsubo } \\
\text { (Apical Ballooning/Stress) } \\
\text { Cardiomyopathy Versus Acute } \\
\text { Myocardial Infarction }\end{array}$ & $\begin{array}{l}\text { Vallabhajosyula et } \\
\text { al. (2020) }\end{array}$ & $\begin{array}{l}\text { Determinar as taxas e tendências de complicações e } \\
\text { mortalidade intra-hospitalar em cardiomiopatia de } \\
\text { Takotsubo e comparar ao infarto agudo do miocárdio } \\
\text { usando um registro nacional dos Estados Unidos }\end{array}$ & $\begin{array}{l}\text { Estados } \\
\text { Unidos }\end{array}$ & $\begin{array}{c}\text { The American Journal } \\
\text { of Cardiology }\end{array}$ \\
\hline $\begin{array}{l}\text { Characteristics and mid-term } \\
\text { follow-up of a single-center } \\
\text { population affected by Tako-Tsubo } \\
\text { cardiomyopathy }\end{array}$ & $\begin{array}{l}\text { Vizzardi et al. } \\
\text { (2015) }\end{array}$ & $\begin{array}{c}\text { Definir características clínicas e instrumentais de uma } \\
\text { população italiana de pacientes com cardiomiopatia } \\
\text { de Takotsubo e relatar seus prognósticos a curto e } \\
\text { médio prazo }\end{array}$ & Itália & $\begin{array}{l}\text { Journal of } \\
\text { Cardiovascular } \\
\text { Medicine }\end{array}$ \\
\hline $\begin{array}{l}\text { Stress-induced cardiomyopathy } \\
\text { (Tako-Tsubo syndrome) in Austria }\end{array}$ & Weihs et al. (2013) & $\begin{array}{l}\text { Coletar dados de pacientes de centros com } \\
\text { instalações intervencionistas para definir mais } \\
\text { completamente a apresentação clínica, eventos de } \\
\text { estresse subjacentes e diferentes tipos de } \\
\text { anormalidades de movimento da parede, bem como o } \\
\text { prognóstico a longo prazo desta síndrome }\end{array}$ & Áustria & $\begin{array}{l}\text { European Heart } \\
\text { Journal: Acute } \\
\text { Cardiovascular Care }\end{array}$ \\
\hline $\begin{array}{l}\text { Management of Takotsubo } \\
\text { cardiomyopathy in non-academic } \\
\text { hospitals in France: The } \\
\text { Observational French SyndromEs of } \\
\text { TakoTsubo (OFSETT) study }\end{array}$ & Yayehd et al. (2015) & $\begin{array}{c}\text { Fornecer uma descrição extensa dos pacientes } \\
\text { internados no hospital por cardiomiopatia de } \\
\text { Takotsubo na França e estudar o manejo e os } \\
\text { prognósticos desses pacientes }\end{array}$ & França & $\begin{array}{l}\text { Archives of } \\
\text { Cardiovascular } \\
\text { Diseases }\end{array}$ \\
\hline $\begin{array}{l}\text { Impact of triggering event in } \\
\text { outcomes of stress-induced } \\
\text { (Takotsubo) cardiomyopathy }\end{array}$ & Yerasi et al. (2016) & $\begin{array}{l}\text { Descrever a experiência com a síndrome de } \\
\text { Takotsubo e, em particular, examinar o } \\
\text { efeitos do gatilho subjacente sobre os prognósticos }\end{array}$ & $\begin{array}{l}\text { Estados } \\
\text { Unidos }\end{array}$ & $\begin{array}{l}\text { European Heart } \\
\text { Journal: Acute } \\
\text { Cardiovascular Care }\end{array}$ \\
\hline
\end{tabular}

Fonte: Autores.

\section{Discussão}

\section{Perfil dos pacientes: epidemiologia, gatilhos e apresentação clínica}

Observou-se um padrão do perfil dos pacientes no presente estudo sobre a cardiomiopatia do estresse. Os pacientes eram predominantemente mulheres, com idade média entre 60 a 70 anos em mais de 90\% dos casos e apresentavam semelhanças à SCA, com elevados níveis de troponina $\mathrm{T}$ e dos demais marcadores citonecróticos miocárdicos, associados à sintomas como dor torácica e dispneia na maioria dos casos (Ahmed et al., 2012; Misumida et al., 2019; Núñez Gil et al., 2015; Templin et al., 2015; Yayehd et al., 2015).

Em algumas observações, notou-se também que a fração de ejeção do ventrículo esquerdo (VE) estava severamente reduzida em metade dos pacientes, descartando-se, entretanto, o diagnóstico de SCA com a observação de normalidade nas artérias coronárias, por meio da submissão à angiografia coronariana, da história clínica marcada por um fator estressor e da observação por exame de imagem do padrão típico da CT, que é uma protuberância ao lado esquerdo do ápice ventricular com uma base hipercontrátil (Laghari et al., 2014; Vizzardi et al., 2015).

Quanto ao fator estressor, trata-se de um achado clínico crucial para o diagnóstico da CT, havendo, porém, bastante divergência dos pacientes observados nos estudos analisados. Nesse cenário, constataram-se casos em que fatores estressantes físicos, como êmese, queda, exercícios físicos de alta intensidade, derrame recente, pericardiocentese e colecistectomia como os principais desencadeadores da CT (Kim et al., 2018; Weihs et al., 2013). Entretanto, ao analisar pacientes do sexo feminino durante pós-menopausa, que são o principal grupo de risco acometido pela CT, os gatilhos emocionais eram mais comuns, enquanto gatilhos físicos foram mais prevalentes entre homens (Meissner et al., 2011; Samardhie et al., 2011; Templin et al., 2015). Contudo, apesar de todas essas informações, não foi observado um gatilho emocional comum entre os pacientes com 
uma proporção significativa (Yerasi et al., 2016).

No eletrocardiograma (ECG), são raros os casos em que o paciente não apresentou alterações, em que se destacam casos com supradesnivelamento do segmento ST como principal alteração, com ondas T gigantes negativas, com segmento ST em depressão e com inversão da onda T (Fröhlich et al., 2011). Já quanto aos fatores de risco, a hipertensão arterial é mais prevalente seguido pela diabetes mellitus, pelo tabagismo e por um histórico de IAM ou histórico de doença cardiovascular na família (Lee et al., 2017; Weihs et al., 2013).

Ademais, observou-se que se trata de uma doença com baixa prevalência, com cerca de 1,7 a 2,2\%, caracterizada como diagnóstico diferencial da SCA, não havendo ainda um consenso sobre a patogênese da CT. (Dias et al., 2013; Eitel et al., 2011; Yerasi et al., 2016). Assim, dá-se destaque para a necessidade da coleta do perfil clínico alargado para diagnóstico e tratamento corretos dessa condição (Eitel et al., 2011).

\section{Diagnóstico}

Devido às diversas semelhanças entre suas apresentações clínicas e laboratoriais, a Cardiomiopatia de Takotsubo é um importante diagnóstico diferencial do infarto agudo do miocárdio, seja este com ou sem presença de supradesnivelamento do segmento ST (Fröhlich et al., 2011). Uma importante peculiaridade da CT é que as artérias coronárias não costumam apresentar lesões e as anormalidades dos segmentos ventriculares tendem a ser resolvidas dentro de alguns dias ou de semanas (Núñez Gil et al., 2015). Sendo assim, esse quadro, na maioria das vezes, possui um prognóstico mais positivo em relação às doenças isquêmicas cardíacas e, por conta disso, a CT é definitivamente diagnosticada apenas após a exclusão de alguma síndrome coronariana aguda (Misumida et al., 2019).

A Cardiomiopatia de Takotsubo torna-se um diagnóstico mais provável quando os biomarcadores, principalmente o nível sérico de enzimas cardíacas, demonstram um aumento leve em contraste ao grave comprometimento hemodinâmico apresentado no paciente (Laghari et al., 2014). Contudo, como aponta o estudo de Templin et al. (2015), os níveis de troponina e as alterações eletrocardiográficas na admissão não são suficientes para diferenciar Cardiopatia de Takotsubo de síndrome coronariana aguda. Nessa pesquisa, $87 \%$ dos pacientes com essa cardiomiopatia apresentaram níveis de troponina elevados e quase $80 \%$ possuíam sinais de isquemia miocárdica no ECG. Portanto, a angiografia coronária precoce permanece necessária para descartar adequadamente a ocorrência de uma síndrome coronária aguda (Templin et al., 2015; Weihs et al., 2013).

Outro exame de grande importância para o rastreamento da CT é a ecocardiografia, por meio da qual é possível avaliar a função ventricular e a presença de anormalidades reversíveis do movimento da parede (ARMP), características típicas dessa cardiopatia (Looi et al., 2020; Weihs et al., 2013). Dessa forma, é possível observar os padrões de acinesia, hipocinesia e discinesia; e tipo de balonismo ventricular apresentado (Weihs et al., 2013). É importante que a ecocardiografia seja realizada precocemente, uma vez que as disfunções típicas podem se recuperar rapidamente (Looi et al., 2020)

O diagnóstico é plenamente estabelecido quando se constata, por meio dos exames complementares previamente citados, que os pacientes apresentam os critérios de Mayo atualizados para cardiomiopatia de Takotsubo. Esses são: hipocinesia, acinesia ou discinesia transitórias dos segmentos médios do ventrículo esquerdo com ou sem envolvimento apical; anormalidades regionais do movimento da parede estendendo-se para uma parede além da área irrigada por única artéria coronária; a ausência de obstrução coronária doença ou de evidência angiográfica de ruptura aguda de placa ateromatosa; aparecimento de novas anormalidades eletrocardiográficas (elevação do segmento ST e/ou inversão da onda T) ou uma leve elevação da troponina cardíaca; e a ausência de feocromocitoma ou de miocardite (Samardhie et al., 2011; Yayehd et al., 2015).

Ademais, a presença de um fator desencadeante, como estresse físico e/ou emocional, é um componente essencial na cardiomiopatia induzida por estresse, estando presente na maioria dos casos (Laghari et al., 2014; Núñez Gil et al., 2015; 
Yerasi et al., 2016). Sendo assim, a investigação desse componente na história do paciente também é de grande importância para o diagnóstico correto dessa cardiopatia.

Também é importante ressaltar ferramentas que, no futuro, podem possuir importante papel no diagnóstico da Cardiopatia de Takotsubo. Em relação a isso, o estudo de Jaguszewski et al. (2014) descreveu, pela primeira vez, uma assinatura de microRNAs circulantes no plasma que podem servir futuramente como marcadores sensíveis e específicos para o diagnóstico de Cardiomiopatia de Takotsubo durante sua fase aguda. Com isso, seria possível diferenciar a CT de casos dos de infarto agudo do miocárdio com supradesnivelamento do segmento ST.

Além disso, o estudo de Nascimento et al. (2014) buscou avaliar a utilidade de um novo parâmetro para a diferenciação entre a CT e o infarto agudo do miocárdio com supradesnivelamento do segmento ST (IAMCST). Essa medida é denominada TEFP (do inglês, Troponin-Ejection Fraction Product) e refere-se ao produto entre o nível de troponina sérica e o valor da fração de ejeção ventricular esquerda, sendo aplicada em pacientes críticos hospitalizados. Essa pesquisa de Nascimento et al. (2014) constatou o TEFP como uma ferramenta simples, mas com capacidade precisa na diferenciação entre CT e IAMCST. Segundo os estudos, quando esse parâmetro possuiu valor maior ou igual a 250 , houve $88 \%$ de valor preditivo positivo para IAMCST, enquanto que em medidas menores que 250 , houve $95 \%$ de valor preditivo negativo para IAMCST (Nascimento et al., 2014).

\section{Manejo e tratamento}

A escolha do local de internação é uma das dificuldades encontradas no manejo de pacientes portadores da cardiomiopatia de Takotsubo, pois muitas vezes o quadro pode se agravar e a necessitar de resposta imediata, a transferência prejudica a resposta rápida a complicações (Samardhie et al., 2011). Segundo o artigo de Núñez Gil et al. (2015), um estudo retrospectivo, comorbidades pré existentes, como ataques de asma, embolia pulmonar, cirurgias e doença cerebrovascular, foram considerados importantes modificadores do prognóstico desses pacientes, assim, na dúvida de onde internar o paciente a ausência de comorbidades resulta em um aumento na segurança da admissão em uma enfermaria de hospital.

Os pacientes com quadro inicial da cardiomiopatia de Takotsubo aguda recebem com frequência manejo e tratamento para SCA e/ou tratamento de apoio para insuficiência cardíaca congestiva (ICC) (Eitel et al., 2011). O fármaco mais frequentemente utilizado nos estudos analisados foi o ácido acetilsalić́lico, mais de $90 \%$ dos casos, seguido de clopidogrel em cerca de $70 \%$ dos casos, além de heparina de baixo peso molecular, betabloqueadores, inibidores da enzima conversora de angiotensina (IECA), vasodilatadores e diuréticos (Eitel et al., 2011; Weihs et al., 2013; Yayehd et al., 2015).

A utilização da terapia de suporte com medicações direcionada ao tratamento de SCAs e ICC nos pacientes portadores da cardiomiopatia de Takotsubo, mostra-se eficaz para o manejo inicial, ocasionando a recuperação da função ventricular (Eitel et al., 2011; Laghari et al., 2014). Em um estudo descritivo de série de casos, os pacientes que sobreviveram a essa etapa inicial aguda, obtiveram sua função ventricular restabelecida de 1 a 4 semanas após o início do tratamento (Laghari et al., 2014).

De acordo com Kim et al. (2018), o benefício da profilaxia sobre a mortalidade apresenta-se controverso, já que o uso desses fármacos não afetou o desenvolvimento, prognóstico e recorrência da doença. Entretanto, em um estudo realizado na universidade de Zurich em colaboração com 25 centros cardiovasculares em 9 países, a utilização de fármacos IECA no tratamento da cardiomiopatia de Takotsubo foram associados à melhora da sobrevida em 1 ano, embora não tenham encontrado evidências de qualquer benefício para o uso de beta-bloqueadores (Templin et al., 2015).

\section{Complicações, prognósticos e mortalidade}

As complicações com maior predominância em pessoas acometidas pela CT vão desde a intercorrências cardíacas 
como insuficiência cardíaca, arritmias, complicações iatrogênicas, insuficiência renal, até outras derivadas de comorbidades como hipertensão e Diabetes Mellitus (Núñez Gil et al., 2015). Segundo os estudos de Santoro et al. (2019), a taxa de complicações dos pacientes analisados com CT foi de $23,4 \%$. Ademais, pacientes que apresentam respostas de catecolaminas desproporcionalmente aumentadas em situações de estresse e sensibilidade simpática cardíaca elevada tendem a mostrar piora clínica (Dias et al., 2013; Ghadri et al., 2018).

Em seus estudos, Samardhie et al. (2011) relataram que 21\% dos pacientes internados apresentaram complicações, tais como choque cardiogênico e edema agudo de pulmão, levando a necessidade de ventilação mecânica e Unidade de terapia intensiva (UTI). Já Laghari et al. (2014) observaram as mesmas complicações juntamente com insuficiência cardíaca em 48,27\% dos pacientes, mostrando uma elevada taxa de pacientes com complicações raras. Nesses pacientes, a terapia preferencial foi a bomba de balão intra-aórtico (BIA), além da ventilação mecânica como suporte (Laghari et al., 2014).

A literatura apresentou taxa de mortalidade heterogênea, demonstrando que ainda há incertezas nos resultados apresentados. O estudo de Kim et al. (2018) observou que 34\% dos pacientes vieram a óbito até 8 anos após o diagnóstico. Essa alta taxa estava diretamente associada a doenças como câncer e outras comorbidades, além do estresse físico e emocional (Eitel et al., 2011; Frangieh et al., 2016). Outros estudos também demonstraram o câncer, diabetes mellitus, o sexo masculino, bem como doenças severas associadas como fatores importantes na evolução, complicações e na mortalidade da CT, mostrando aumento importante na mortalidade na fase aguda da doença (Jaguszewski et al., 2014; Laghari et al., 2014; Stiermaier et al., 2016; Vallabhajosyula et al., 2020; Weihs et al., 2013).

Apesar do prognóstico preocupante em pacientes com comorbidades e doenças severas associadas, a CT apresenta, em vários estudos, taxa de mortalidade inferior às apresentadas acima. O artigo de Yerasi et al. (2016) apresenta uma taxa de 3\% de mortes em pacientes sem doenças associadas corroborando o estudo de Eitel et al. (2011), que apregoa que apesar da gravidade aguda da doença, as complicações costumam ser raras e o prognóstico dos pacientes favorável.

Segundo os estudos de Schwarz et al. (2017), os pacientes com CT evoluíram de forma mais lenta do que era esperado, apresentando processo de fibrose que se desenvolve posteriormente a edema de miocárdio, podendo indicar que o acometimento pela CT pode não ser totalmente reversível.

Em síntese, embora a taxa de mortalidade seja variável, os dados apresentados sugerem que a CT é o reflexo de uma síndrome subclínica, e que a piora do seu prognóstico ou o aumento da sua fatalidade está direta ou indiretamente ligada a uma comorbidade subjacente (Kim et al., 2018; Weihs et al., 2013; Yerasi et al., 2016).

\section{Comparação com a Síndrome Coronariana Aguda}

A CT, como já citado anteriormente, apresenta muitas semelhanças clínicas com a SCA, o que se apresenta como um desafio na diferenciação entre as duas. Os quadros clínicos se assemelham pela presença de dor torácica e dispneia, incluindo alterações no ECG e nos biomarcadores cardíacos (Ghadri et al., 2016; Samardhie et al., 2011). Além disso, podem apresentar dados de mortalidade e complicações intra-hospitalares semelhantes (Samardhie et al., 2011).

As alterações eletrocardiográficas, embora presente em ambas as condições, apresentam padrões distintos. O prolongamento do QTc é um achado comum na CT, enquanto a depressão do segmento ST é mais comumente encontrada na SCA (Ghadri et al., 2016). Em um estudo de coorte realizado apenas com mulheres, notou-se que a inversão da onda T generalizada foi mais frequentemente observada em pacientes com CT, apresentando-se em 57\% dos pacientes, em comparação com $10 \%$ dos pacientes com SCA. Além disso, a inversão da onda T sem depressão ST no primeiro dia de internação esteve fortemente relacionado a CT, revelando um potencial discriminatório do ECG em CT (Looi et al., 202).

Outros achados eletrocardiográficos úteis na diferenciação de CT e SCA, foram o intervalo PQ mais longo e a largura do complexo QRS menor em pacientes com CT, assim como elevação do segmento ST em aVR mais prevalente em 
CT (31\%) do que em SCA (3\%) (Frangieh et al., 2016). A depressão de ST esteve comumente em todas as derivações de ECG em pacientes com SCA, exceto aVR (Frangieh et al., 2016; Ghadri et al., 2016). Além disso, a elevação de ST em paciente com CT também esteve associada à presença de gatilho emocional, como pânico, medo e ansiedade (Frangieh et al., 2016).

Em relação aos biomarcadores cardíacos, alguns estudos concluíram que a troponina encontra-se elevada nos dois grupos, não sendo o melhor biomarcador diferenciador (Cai et al., 2017; Ghadri et al., 2016; Jaguszewski et al., 2014; Meissner et al., 2011). Porém, em comparação com SCA, observou-se a Troponina e o peptídeo natriurético mais elevados na admissão em pacientes com CT e uma elevação da creatina quinase maior em pacientes com SCA (Ghadri et al., 2016). Além disso, o pico nos níveis de troponina divergiu, alcançando valores menores em pacientes com CT (Frangieh et al., 2016; Meissner et al., 2011).

Nas análises de Meissner et al. (2011), um estudo comparativo, concluiu-se que a copeptina possui um potencial útil para distinguir SCA de CT, em que os níveis de copeptina eram consideravelmente mais baixos em pacientes com CT. Dessa forma, aliada a outros escores, a copeptina se apresentou com maior acurácia diagnóstica quando comparada aos níveis de troponina.

Em comparação à apresentação clínica, a CT possui algumas características mais prevalentes, acometendo majoritariamente mulheres em idade pós menopausa que apresentaram gatilhos estressantes, seja físico ou mental (Dias et al., 2013; Vallabhajosyula et al., 2019). A presença desse desencadeante emocional se apresentou nos estudos como um aspecto marcante da CT (Ghadri et al., 2016; Meissner et al., 2011). Um outro fator importante foi a presença de uma doença neurológica ou psiquiátrica mais prevalente na CT em comparação a SCA, apresentando-se em até o dobro dos casos, o que necessita de maiores estudos para associação como um fator de risco relevante (Ghadri et al., 2016; Medeiros et al., 2014; Templin et al., 2015).

Por fim, em relação ao acometimento cardíaco, a fração de ejeção do ventrículo esquerdo na admissão era consideravelmente menor em pacientes com CT em comparação com pacientes com SCA (Frangieh et al., 2016; Meissner et al., 2011; Templin et al., 2015). Além disso, na análise de ecocardiogramas transtorácicos a CT apresentou padrões de anormalidades no movimento da parede do ventrículo esquerdo, além do acometimento anormal transitório do ventrículo direito, esses achados podem ser úteis na diferenciação por imagem (Cai et al., 2017).

\section{Conclusão}

Baseando-se nos aspectos observados e discutidos previamente, foi possível a compreensão da apresentação clínica que identifica a CT. Assim, de modo geral, a CT é uma afeç̧ão que acomete predominantemente mulheres que apresentam fatores estressantes físicos ou mentais e deve ser investigada como um diagnóstico diferencial da SCA e, por possuir um prognóstico geralmente melhor, deve ser pesquisada somente após exclusão da SCA.

Outrossim, apesar da CT e da SCA apresentarem clínica semelhante, destaca-se como método de diferenciação o eletrocardiograma e o ecocardiografia para análise dos critérios de Mayo, que se contemplados confirmam a CT. Ainda, quanto aos biomarcadores, nota-se a Troponina com níveis elevados semelhantes em ambas e outros biomarcadores, como a Copeptina, só apresentaram valor distintivo quando associados a outros parâmetros.

Ademais, constatou-se que a manifestação aguda da CT possui gravidade semelhante a SCA, porém, no que diz respeito a evolução dos pacientes, apesar da ausência de um valor consistente entre os autores, ficou notável o pior prognóstico e maior mortalidade intimamente relacionados com a presença de comorbidades. Dessa forma, a associação de outras doenças que aumentam os riscos de complicações é responsável por gerar uma maior dificuldade de alocar o paciente para ser internado.

Ressalta-se ainda a limitação nos dados de mortalidade, devido à dificuldade encontrada por muitos autores dos 
trabalhos analisados em acompanhamento a longo prazo dos pacientes e também a existência de uma fragilidade metodológica na comparação entre a CT e a SCA, devido à maior proporção de mulheres com a CT. Adjunto a isso, para trabalhos futuros, sugere-se que sejam realizados mais estudos primários direcionados à investigação da fisiopatologia e da relação com os gatilhos de estresse, pois foi notada uma escassez dessa abordagem na literatura.

Conclui-se, assim, que as duas afecções, a cardiomiopatia de Takotsubo e a Síndrome Coronariana Aguda, possuem apresentações clínicas bastante semelhantes, devendo a primeira ser investigada como diagnóstico diferencial da segunda. Para a diferenciação das duas e confirmação diagnóstica da cardiomiopatia de Takotsubo, os critérios de Mayo devem ser contemplados, através do auxílio dos exames complementares eletrocardiograma e ecocardiografia.

Sugere-se assim, que sejam realizados mais estudos que abordam dados relacionados a mortalidade e evolução dos pacientes com Síndrome de Takotsubo, além de estudos que investiguem mais a fundo a fisiopatologia dessa doença e a relação do acometimento da síndrome com seus gatilhos desencadeantes de estresse, pois foram notadas escassez dessa abordagem e divergências literárias.

\section{Referências}

Ahmed, K. A., Madhavan, M., \& Prasad, A. (2012). Brain natriuretic peptide in apical ballooning syndrome (Takotsubo/stress cardiomyopathy): comparison with acute myocardial infarction. Coronary artery disease, 23(4), 259-264.

Botelho, L. L. R., de Almeida Cunha, C. C., \& Macedo, M. (2011). O método da revisão integrativa nos estudos organizacionais. Gestão e sociedade, 5(11), 121-136.

Cai, L., Addetia, K., Medvedofsky, D., \& Spencer, K. T. (2017). Myocardial strain may be useful in differentiating Takotsubo cardiomyopathy from left anterior descending coronary artery ischemia. International journal of cardiology, 230, 359-363.

Dias, A., Franco, E., Mercedes, A., Hebert, K., Messina, D., \& Quevedo, H. C. (2013). Clinical features of takotsubo cardiomyopathy-a single-center experience. Cardiology, 126(2), 126-130.

Eitel, I., von Knobelsdorff-Brenkenhoff, F., Bernhardt, P., Carbone, I., Muellerleile, K., Aldrovandi, A., Francone, M. et al. (2011). Clinical characteristics and cardiovascular magnetic resonance findings in stress (takotsubo) cardiomyopathy. Jama, 306(3), 277-286.

Frangieh, A. H., Obeid, S., Ghadri, J. R., Imori, Y., D'Ascenzo, F., Kovac, M., Ruschitzka, M. et al. (2016). ECG criteria to differentiate between Takotsubo (stress) cardiomyopathy and myocardial infarction. Journal of the American Heart Association, 5(6), e003418.

Fröhlich, G. M., Schoch, B., Schmid, F., Keller, P., Sudano, I., Lüscher, T. F., Noll, G. et al. (2012). Takotsubo cardiomyopathy has a unique cardiac biomarker profile: NT-proBNP/myoglobin and NT-proBNP/troponin T ratios for the differential diagnosis of acute coronary syndromes and stress induced cardiomyopathy. International journal of cardiology, 154(3), 328-332.

Ghadri, J. R., Cammann, V. L., Jurisic, S., Seifert, B., Napp, L. C., Diekmann, J., Bataiosu, D. R. et al. (2016). A novel clinical score (InterTAK Diagnostic Score) to differentiate takotsubo syndrome from acute coronary syndrome: results from the International Takotsubo Registry. European journal of heart failure, 19(8), 1036-1042.

Ghadri, J. R., Kato, K., Cammann, V. L., Gili, S., Jurisic, S., Di Vece, D., Candreva, A. et al. (2018). Long-term prognosis of patients with Takotsubo syndrome. Journal of the American College of Cardiology, 72(8), 874-882.

Jaguszewski, M., Osipova, J., Ghadri, J. R., Napp, L. C., Widera, C., Franke, J., Fijalkowski, M. et al. (2014). A signature of circulating microRNAs differentiates takotsubo cardiomyopathy from acute myocardial infarction. European heart journal, 35(15), 999-1006.

Kim, H., Senecal, C., Lewis, B., Prasad, A., Rajiv, G., Lerman, L. O., \& Lerman, A. (2018). Natural history and predictors of mortality of patients with Takotsubo syndrome. International journal of cardiology, 267, 22-27.

Laghari, A. H., Khan, A. H., \& Kazmi, K. A. (2014). Takotsubo cardiomyopathy: ten year experience at a tertiary care hospital in Pakistan. BMC research notes, 7(1), 1-5.

Lee, S. R., Lee, S. E., Rhee, T. M., Park, J. J., Cho, H., Lee, H. Y., Choi, D. J. et al. (2017). Discrimination of stress (Takotsubo) cardiomyopathy from acute coronary syndrome with clinical risk factors and coronary evaluation in real-world clinical practice. International journal of cardiology, $235,154-161$.

Looi, J. L., Poppe, K., Lee, M., Gilmore, J., Webster, M., To, A., \& Kerr, A. J. (2020). A Score to differentiate Takotsubo syndrome from non-ST-elevation myocardial infarction in women at the bedside. Open heart, 7(1), e001197.

Medeiros, K., O’Connor, M. J., Baicu, C. F., Fitzgibbons, T. P., Shaw, P., Tighe, Zile, M. R. et al. (2014). Systolic and diastolic mechanics in stress cardiomyopathy. Circulation, 129(16), 1659-1667.

Meissner, J., Nef, H., Darga, J., Kovacs, M., Weber, M., Hamm, C., Möllmann, H. et al. (2011). Endogenous stress response in Tako-Tsubo cardiomyopathy and acute myocardial infarction. European journal of clinical investigation, 41(9), 964-970. 
Misumida, N., Ogunbayo, G. O., Kim, S. M., Abdel-Latif, A., Ziada, K. M., \& Sorrell, V. L. (2019). Clinical outcome of Takotsubo cardiomyopathy diagnosed with or without coronary angiography. Angiology, 70(1), 56-61.

Nascimento, F. O., Yang, S., Larrauri-Reyes, M., Pineda, A. M., Cornielle, V., Santana, O., Heimowitz, T. B. et al. (2014). Usefulness of the troponin-ejection fraction product to differentiate stress cardiomyopathy from ST-segment elevation myocardial infarction. The American journal of cardiology, 113(3), 429433.

Núñez Gil, I. J. N., Andrés, M., Delia, M. A., Sionis, A., Martín, A., Bastante, T., Soriano, J. G. C., Vicente, J. A. L. et al. (2015). Characterization of Takotsubo Cardiomyopathy in Spain: Results from the RETAKO National Registry. Revista Española de Cardiología. 68(6), 505-512.

Reis, J. G. V., \& Rosas, G. (2010). Cardiomiopatia de Takotsubo: um diagnóstico diferencial da síndrome coronariana aguda: revisão da literatura. Rev Med Minas Gerais, 20(4), 594-600.

Samardhi, H., Raffel, O. C., Savage, M., Sirisena, T., Bett, N., Pincus, M., Small, A. et al. (2012). Takotsubo cardiomyopathy: an Australian single centre experience with medium term follow up. Internal medicine journal, 42(1), 35-42.

Santoro, F., Gil, I. J. N., Stiermaier, T., El-Battrawy, I., Guerra, F., Novo, G., Guastafierro, F. et al. (2019). Assessment of the German and Italian stress cardiomyopathy score for risk stratification for in-hospital complications in patients with Takotsubo syndrome. JAMA cardiology, 4(9), 892-899.

Santos, C. M. D. C., Pimenta, C. A. D. M., \& Nobre, M. R. C. (2007). The PICO strategy for the research question construction and evidence search. Revista latino-americana de enfermagem, 15(3), 508-511.

Schwarz, K., Ahearn, T., Srinivasan, J., Neil, C. J., Scally, C., Rudd, A., Jagpal, B. et al. (2017). Alterations in cardiac deformation, timing of contraction and relaxation, and early myocardial fibrosis accompany the apparent recovery of acute stress-induced (takotsubo) cardiomyopathy: an end to the concept of transience. Journal of the American Society of Echocardiography, 30(8), 745-755.

Souza, M. T. D., Silva, M. D. D., \& Carvalho, R. D. (2010). Integrative review: what is it? How to do it? Einstein, 8(1), $102-106$.

Stiermaier, T., Moeller, C., Oehler, K., Desch, S., Graf, T., Eitel, C., Vonthein, R. et al. (2016). Long-term excess mortality in takotsubo cardiomyopathy: predictors, causes and clinical consequences. European journal of heart failure, 18(6), 650-656.

Takahashi, J., Saheki, Y., Gardim, S. (2014). O que é PICO e Pico? SlideShare. https://pt.slideshare.net/bibliotecaee/o-que-pico-e-pico.

Templin, C., Ghadri, J. R., Diekmann, J., Napp, L. C., Bataiosu, D. R., Jaguszewski, M., Cammann, V. L. (2015). Clinical features and outcomes of Takotsubo (stress) cardiomyopathy. New England Journal of Medicine, 373(10), 929-938.

Vallabhajosyula, S., Barsness, G. W., Herrmann, J., Anavekar, N. S., Gulati, R., \& Prasad, A. (2020). Comparison of Complications and In-Hospital Mortality in Takotsubo (Apical Ballooning/Stress) Cardiomyopathy Versus Acute Myocardial Infarction. The American Journal of Cardiology, 132, 29-35.

Vallabhajosyula, S., Dunlay, S. M., Murphree, D. H., Barsness, G. W., Sandhu, G. S., Lerman, A., \& Prasad, A. (2019). Cardiogenic shock in takotsubo cardiomyopathy versus acute myocardial infarction: an 8-year national perspective on clinical characteristics, management, and outcomes. JACC: Heart Failure, 7(6), 469-476.

Vizzardi, E., Bonadei, I., Rovetta, R., Sciatti, E., D’Aloia, A., Pezzali, N., Salghetti, F. et al. (2015). Characteristics and mid-term follow-up of a single-center population affected by Tako-Tsubo cardiomyopathy. Journal of Cardiovascular Medicine, 16(5), 326-330.

Weihs, V., Szücs, D., Fellner, B., Eber, B., Weihs, W., Lambert, T., Metzler, B. et al. (2013). Stress-induced cardiomyopathy (Tako-Tsubo syndrome) in Austria. European Heart Journal: Acute Cardiovascular Care, 2(2), 137-146.

Yayehd, K., N'kenon, W., Belle, L., Bataille, V., Hanssen, M., Leddet, P., Aupetit, J. F. et al. (2016). Management of Takotsubo cardiomyopathy in nonacademic hospitals in France: the Observational French SyndromEs of TakoTsubo (OFSETT) study. Archives of cardiovascular diseases, 109(1), 4-12.

Yerasi, C., Koifman, E., Weissman, G., Wang, Z., Torguson, R., Gai, J., Lindsay, J. et al. (2017). Impact of triggering event in outcomes of stress-induced (Takotsubo) cardiomyopathy. European Heart Journal: Acute Cardiovascular Care, 6(3), 280-286. 